\title{
Calcium Control of Triphasic Hippocampal STDP
}

\author{
Daniel Bush* and Yaochu Jin \\ Department of Computing, University of Surrey, Guildford, GU2 7XH, UK \\ *Corresponding author: DrDanielBush@gmail.com \\ Keywords: Synaptic Plasticity, Calcium, Learning, Memory, Hippocampus
}

\begin{abstract}
Synaptic plasticity is believed to represent the neural correlate of mammalian learning and memory function. It has been demonstrated that changes in synaptic conductance can be induced by approximately synchronous pairings of pre- and post- synaptic action potentials delivered at low frequencies. It has also been established that NMDAr-dependent Calcium influx into dendritic spines represents the critical signal for plasticity induction, and can account for this spike-timing dependent plasticity (STDP) as well as experimental data obtained using other stimulation protocols. However, subsequent empirical studies have delineated a more complex relationship between spike-timing, firing rate, stimulus duration and post-synaptic bursting in dictating changes in the conductance of hippocampal excitatory synapses. It has yet to be established whether the Calcium control hypothesis can account for this more recent data. Here, we present a detailed biophysical model of single dendritic spines on a CA1 pyramidal neuron, describe the NMDAr-dependent Calcium influx generated by different stimulation protocols, and present a parsimonious model of Calcium driven kinase and phosphotase dynamics that dictate transitions between binary synaptic weight states. We demonstrate the manner in which this model can account for various experimental observations of synaptic plasticity and be used to make predictions regarding the dynamics of depolarisation and NMDAr activation generated by STDP protocols as well as the synaptic weight change induced under other experimental conditions. We then discuss how this parsimonious, unified computational model of synaptic plasticity might be utilised to appraise the activity-dependent refinement of neural circuitry induced by more realistic firing patterns.
\end{abstract}




\section{Introduction}

Synaptic plasticity - the process of activity dependent change in synaptic conductance - is widely believed to represent the neural correlate of mammalian learning and memory function [1-3]. Since the first experimental demonstrations of long-term potentiation (LTP) and depression (LTD), a wealth of empirical data regarding the induction, expression and maintenance of synaptic plasticity in different cortical regions has been obtained [4-8]. In spite of the heterogeneity of plasticity mechanisms observed throughout the brain, changes in the strength of excitatory synapses afferent on CA1 pyramidal neurons in the hippocampus represent the best studied form in the mammalian cortex [9-12]. At these synapses, Calcium influx into dendritic spines represents the critical signal for synaptic plasticity induction [13-20]. Large, transient elevations in intracellular $\left[\mathrm{Ca}^{2+}\right]$ generate LTP via the preferential activation of kinase pathways while modest, sustained elevations in intracellular $\left[\mathrm{Ca}^{2+}\right]$ generate LTD via the preferential activation of phosphotase pathways [21-25]. Initially, empirical observations of synaptic plasticity were mediated by tetanic stimulation protocols, with high frequency stimulation (HFS; typically $1 \mathrm{~s}$ of $100 \mathrm{~Hz}$ afferent firing) used to induce LTP and low frequency stimulation (LFS; typically $15 \mathrm{mins}$ of $1 \mathrm{~Hz}$ afferent firing) used to induce LTD [4, 5]. In more recent years, it has also been established that temporally correlated pairs or triplets of pre- and post- synaptic action potentials delivered at low frequencies can induce bidirectional spike-timing dependent plasticity (STDP) depending, among other parameters, on their exact temporal offset over a range of $\sim 100 \mathrm{~ms}$ [26-30]. STDP has been examined in a variety of cortical regions and species, and its discovery has both accompanied and accelerated a move in computational neuroscience from rate to temporally coded models of cognitive processing [31, 32].

Early studies of STDP, primarily carried out in hippocampal cell cultures, delineated a straightforward relationship between the relative timing of single pre- and post- synaptic action potentials and subsequent changes in synaptic strength [Figure 1a; 26, 27]. However, more recent examinations using acute hippocampal slices have been unable to induce bidirectional plasticity with pairs of single preand post- synaptic action potentials under standard recording conditions [Figure 1b; 28-30, 33, 34]. These results suggest a more complex picture, where synaptic plasticity is dependent not just on relative spike timing, but also on the frequency, duration and nature of spike pairings - with a triphasic STDP curve obtained at CA3-CA1 synapses only when pairings are delivered at approximately theta frequency $(>5 \mathrm{~Hz})$ and involve multiple post-synaptic spikes [20, 29, 32; Figure 1c]. Similar results have been obtained at excitatory connections between cortical pyramidal neurons [35]. Other experimental data indicates that potentiation and depression events are switch-like transitions between binary conductance states, mediated by kinase and phosphotase pathways that are co-activated and competitive [36-40]. The kinetics of kinase and phosphotase activation also differ significantly, as LTP can be rapidly induced by appropriate patterns of activity while LTD requires prolonged stimulation $[15,17,29]$. Computational modelling of synaptic plasticity has demonstrated that the dynamics of Calcium influx through the NMDA receptor (NMDAr) is sufficient to account for empirical data obtained using multiple stimulation protocols, integrating an array of experimental results within a single theoretical framework [18, 41-48]. However, these models as yet fail to assimilate or replicate various aspects of more recently obtained synaptic plasticity data such as those described above.

Here, we present a revised model in which NMDAr-dependent Calcium influx at individual dendritic spines controls the dynamics of putative kinase and phosophotase pathways according to a modified Hill function inspired by recent advances in systems biology [49, 50]. The local activation of kinase and phosphotase, in turn, dictates the probability of dynamic transitions between binary high and low synaptic conductance states in a stochastic Markov process. We tune the parameters of this model to specifically account for recent observations of triphasic hippocampal STDP, and subsequently demonstrate that it can also replicate observations of synaptic plasticity induced by other stimulation protocols and under regimes of selective pharmacological blockade. We then utilise this model to infer the time course of residual depolarisation in the spine generated by a backpropagating action potential (bAP) and the magnitude of NMDAr-dependent Calcium influx when significant depolarisation follows glutamate binding by $>50 \mathrm{~ms}$; as well as describing the implications of bAP attenuation and stimulation frequency for synaptic plasticity - in each case demonstrating that the model can support recent empirical findings. As such, we provide a comprehensive but parsimonious description of synaptic plasticity at the CA3-CA1 synapse, allowing further simulations to make robust predictions regarding the activity-dependent refinement of neural circuitry induced by more complex and realistic firing patterns without significant computational cost. 


\section{Results}

We examine a detailed biophysical model of multiple dendritic spines that receive pre- and postsynaptic stimulation corresponding to canonical experimental protocols used to induce synaptic plasticity (see Methods). The parameters of the neuron model are matched to recent experimental data obtained at the CA3-CA1 synapse wherever available. Stimulation generates membrane depolarisation via conductance based synapses and backpropagating action potentials, as well as Calcium influx through NMDA receptors (NMDAr- $\left[\mathrm{Ca}^{2+}\right]$ ) at each spine. This Calcium influx dictates the dynamics of putative kinase and phosphotase activity according to modified Hill functions that are inspired by recent advances in systems biology. The activity of kinase and phosphotase pathways, in turn, determine the probability of transitions between binary high and low conductance states in a stochastic Markov process. The resultant change in total afferent synaptic conductance across $N=10000$ synaptic inputs can then be compared to changes in field excitatory post-synaptic potentials (fEPSPs) recorded in empirical studies.

\subsection{Induction of Synaptic Plasticity by Spike-timing Stimulation}

Recent experiments conducted in acute hippocampal slices, which closely approximate the conditions present in vivo, have demonstrated that the plasticity of CA3-CA1 synapses is jointly dependent upon the temporal offset of pre- and post- synaptic firing, number of post-synaptic spikes fired, frequency of spike pairings, and duration of stimulation [28-30]. Firstly, we aim to ascertain whether the Calcium control hypothesis - which has been demonstrated to successfully reproduce earlier STDP data obtained in culture (Figure 1a), as well as that induced by other activity patterns - can be revised to account for this joint dependency [18, 41-48]. The experimental data we aim to replicate can be characterised by considering the effects of two different stimulation protocols - pairing 100 single preand post- synaptic spikes (hereafter referred to as 'spike pairing') at low frequencies $(0.1-5 \mathrm{~Hz})$, which generates a depression-only learning rule (Figure 1b); or pairing a single pre-synaptic spike with two post-synaptic spikes (hereafter referred to as 'triplet pairing'), which generates a triphasic bidirectional learning rule after 100 pairings at a frequency of $5 \mathrm{~Hz}$ (Figure 1c), an unsaturated potentiation-only rule after 30 pairings at $5 \mathrm{~Hz}$ (Figure 1d), or mild depression after 100 pairings at a frequency of $0.5 \mathrm{~Hz}$ (data not shown). Each of these data sets can be fitted by a learning rule composed of Gaussian (or a sum of Gaussian) curves centred at short, positive temporal offsets [29].
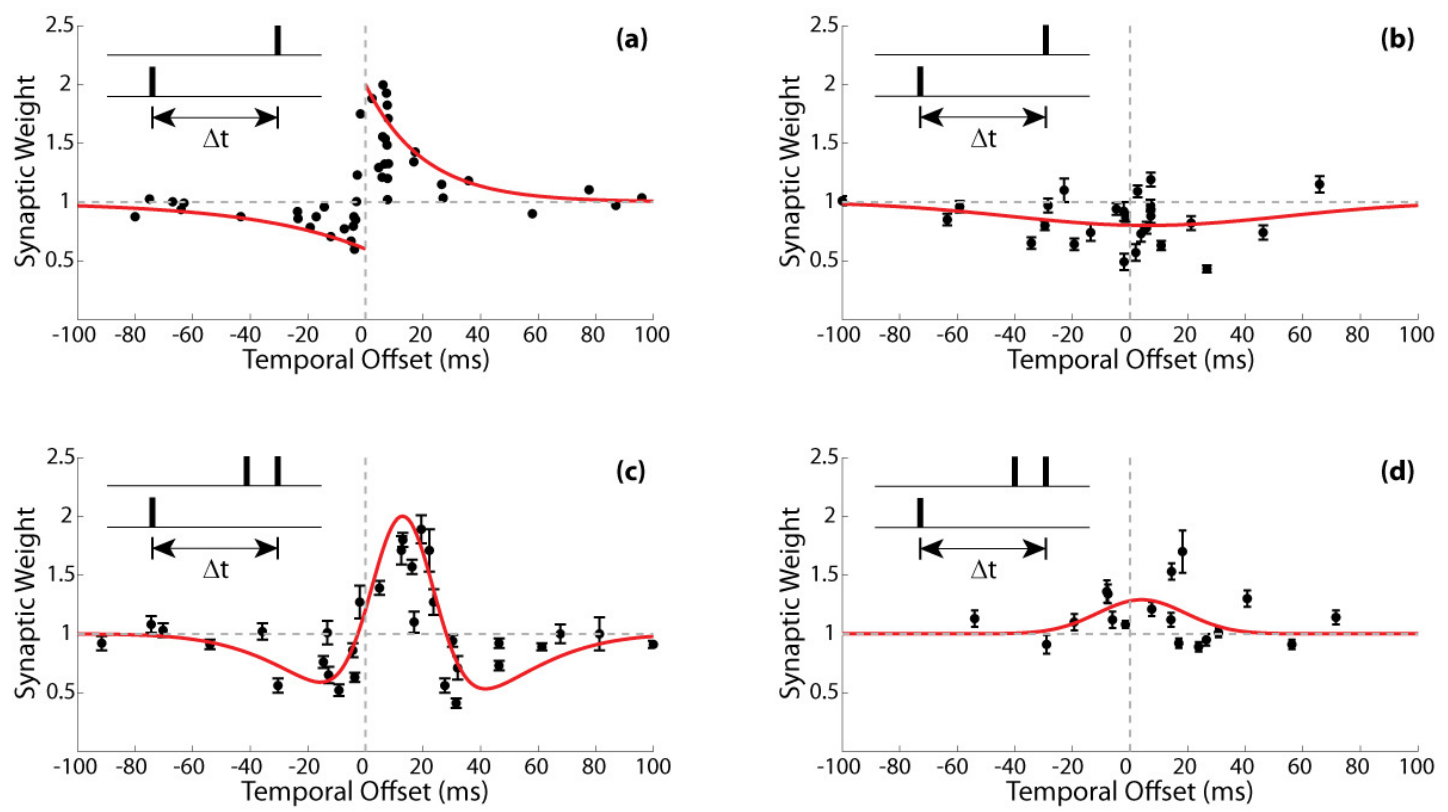

Fig. 1 Summary of the STDP data obtained in hippocampal (a) cell cultures [26] and (b-d) acute slice preparations [29]. (a) The change in EPSP generated by 100 pairs of single pre- and post- synaptic spikes at $1 \mathrm{~Hz}$ and various temporal offsets $\left(\Delta t=t_{\text {post }}-t_{\text {pre }}\right)$ in hippocampal cell cultures and two exponential curves fit to this data (with $\mathrm{A}_{+}=1, \tau_{+}=20 \mathrm{~ms}, \mathrm{~A} .=-0.4$ and $\tau_{-}=40 \mathrm{~ms}$ ) from $\mathrm{Bi}$ and Poo [26]. (b) The change in EPSP generated by 100 pairs of single pre- and post- synaptic spikes at frequencies of $0.1-5 \mathrm{~Hz}$ in acute slice preparations and a Gaussian fit to this data (with $\mu=6 \mathrm{~ms}, \sigma=48 \mathrm{~ms}$ and a peak of 0.8 ) from Wittenberg and Wang [29]. (c) The change in EPSP generated by 100 pairings of single pre-synaptic spikes with two post-synaptic spikes at 5Hz in acute slice preparations and the sum of two Gaussians fit to this data (with $\mu_{1}=15 \mathrm{~ms}, \sigma_{1}=32 \mathrm{~ms}$ and $\mu_{2}=13 \mathrm{~ms}, \sigma_{2}=11 \mathrm{~ms}$ ) from Wittenberg and Wang [29]. (d) The change in EPSP generated by 30 pairings of single pre-synaptic spikes with two postsynaptic spikes at $5 \mathrm{~Hz}$ in acute slice preparations and a Gaussian fit to this data (with $\mu=4 \mathrm{~ms}, \sigma=15 \mathrm{~ms}$ and a peak value of 1.29) from Wittenberg and Wang [29] 
An examination of the simulated NMDAr- $\left[\mathrm{Ca}^{2+}\right]$ dynamics produced by these stimulation protocols illustrates the foundations for the Calcium control hypothesis - high peak values being generated at short, predominantly positive temporal offsets and decaying exponentially with increasing $\Delta \mathrm{t}$ (Figure $2 \mathrm{a}, \mathrm{b})$. Intuitively, setting thresholds for kinase $\left(\theta_{\mathrm{LTP}}\right)$ and phosphotase $\left(\theta_{\mathrm{LTD}}\right)$ activation as the peak NMDAr- $\left[\mathrm{Ca}^{2+}\right]$ values achieved by spike pairings at short and long temporal offsets respectively will generate Gaussian learning curves centred at short positive temporal offsets that reflect empirical data. We utilise these peak NMDAr- $\left[\mathrm{Ca}^{2+}\right]$ values to guide parameterisation of the modified Hill functions that control kinase and phosphotase activity - setting thresholds such that significant kinase activity is only generated by $5 \mathrm{~Hz}$ triplet pairing at short, positive temporal offsets and significant phosphotase activity is only generated by peak NMDAr- $\left[\mathrm{Ca}^{2+}\right]$ values that are greater than the minimum generated by $5 \mathrm{~Hz}$ spike pairings (Figure $2 \mathrm{a}, \mathrm{b}$ ). Hill co-efficients and dissociation constants are set in line with previous experimental and theoretical studies, and the empirically observed competition between these two pathways is accounted for by dictating that kinase activity partially inhibits phosphotase activity [40, 41, 44, 49, 50]. Finally, the activation of kinase and phosphotase must also obey different kinetics, such that LTP can be induced rapidly, by a small number of triplet pairings, while significant LTD requires more sustained stimulation $[15,17,29]$. This is achieved by assigning a much more rapid time constant of decay to the kinase pathway compared to the phosphotase pathway $(50 \mathrm{~ms}$ and $2000 \mathrm{~ms}$ respectively).

By constraining the parameters of the plasticity model in this way, we are able to qualitatively replicate the full array of experimental data described above (Figure 2c-f). Spike pairings delivered at low frequencies $(0.1-5 \mathrm{~Hz})$ generate a depression only learning rule centred at short positive temporal offset $\left(\mu_{D}=22.5 \mathrm{~ms}\right)$ and extending over a wide range of temporal offsets $\left(\sigma_{D}=35.1 \mathrm{~ms}\right)$; while spike triplets delivered at $5 \mathrm{~Hz}$ generate a potentiation only learning rule centred at short positive temporal offset $\left(\mu_{\mathrm{P}}=19.5 \mathrm{~ms}\right)$ with a narrower range $\left(\sigma_{\mathrm{P}}=8.9 \mathrm{~ms}\right)$ when applied for short durations and a triphasic learning rule (with $\mu_{D}=16.2 \pm 67.1 \mathrm{~ms}$ and $\mu_{\mathrm{P}}=19.2 \pm 9.0 \mathrm{~ms}$ ) if applied for longer periods. An examination of Calcium, kinase and phosphotase dynamics during typical potentiating and depressing stimuli illustrates the mechanisms of the plasticity model (Figure 3a-d). Moderate elevations in intracellular Calcium, generated here by acausal $5 \mathrm{~Hz}$ triplet pairings at short temporal offsets, selectively activate the phosphotase pathway and subsequently increase the probability of synapses making the transition from high to low weight states. Conversely, larger elevations in intracellular Calcium, generated here by causal $5 \mathrm{~Hz}$ triplet pairings at short temporal offsets, activate both kinase and phosphotase pathways, the former inhibiting the latter and subsequently increasing the probability of synapses making the transition from low to high weight states.

Following a potentiating or depressing stimulus, the return of kinase and phosphotase activity to equilibrium dictates that the overall distribution of strong and weak synaptic weights returns to its initial state over a time course of $\sim 1$ hour, in correspondence with the decay of early-phase long-term potentiation and depression (E- LTP / LTD) observed empirically [Figure 3e, f; 51-53]. It is also interesting to note that, due to the higher gain and shorter time constant governing kinase activation, transitions between low and high weight states induced by potentiating stimuli take consistently less time than transitions between high and low weight states induced by depressing stimuli $(69 \pm 50 \mathrm{~s}$ at $\Delta \mathrm{t}=15 \mathrm{~ms}$ and $108 \pm 55 \mathrm{~s}$ at $\Delta \mathrm{t}=-15 \mathrm{~ms}$ for $5 \mathrm{~Hz}$ Triplet pairing, for example), and each is on a similar timescale to that observed experimentally $(80 \pm 70$ s for LTP and $183 \pm 126$ s for LTD in O'Connor, Wittenberg and Wang [37]; 38s for LTP in Bagal et al. [39]). 

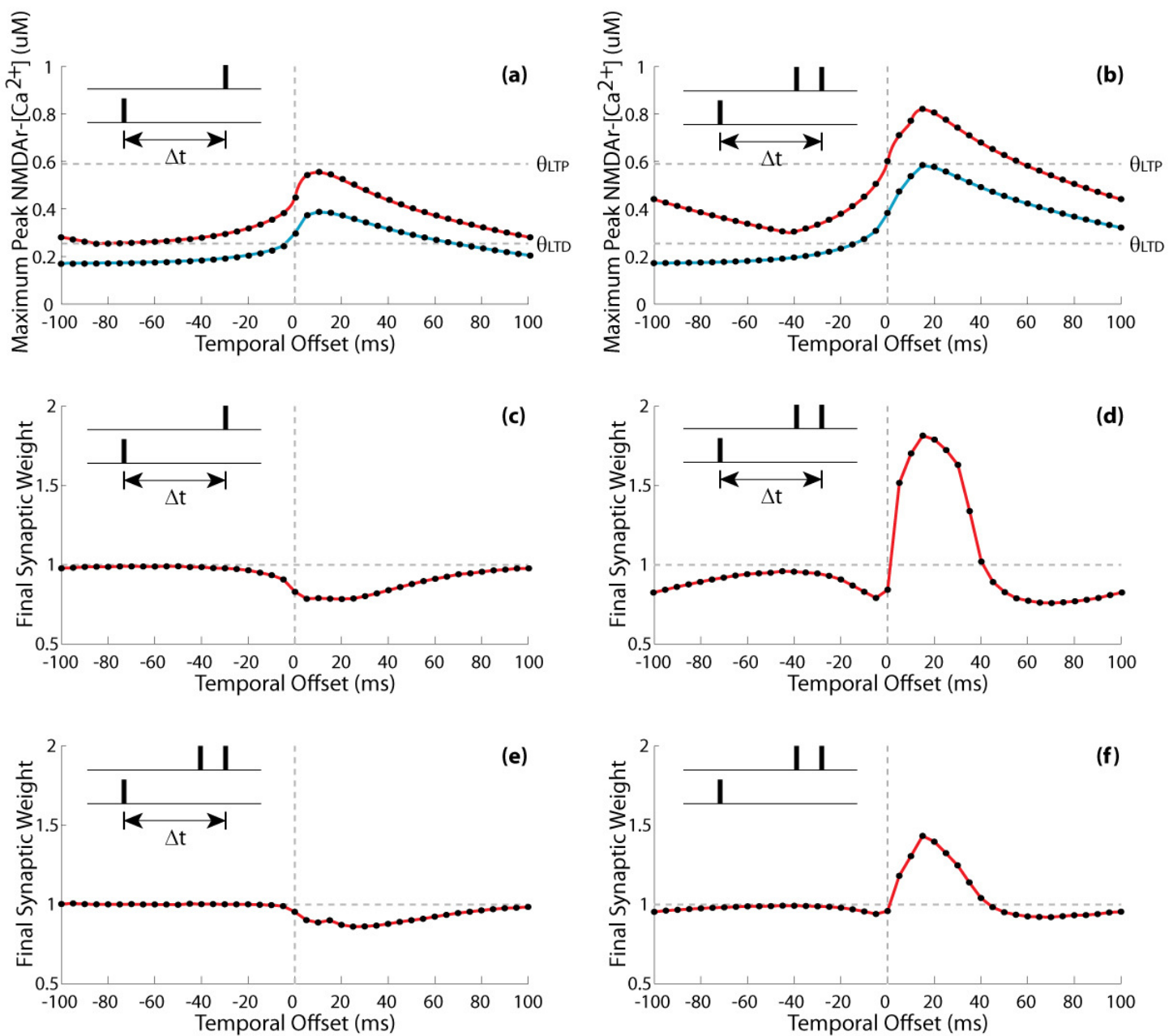

Fig. 2 NMDAr- $\left[\mathrm{Ca}^{2+}\right]$ and synaptic weight change generated by spike and triplet pairing stimulation protocols. (a) Maximum peak NMDAr- $\left[\mathrm{Ca}^{2+}\right]$ generated by 100 spike pairings delivered at $0.5 \mathrm{~Hz}$ (blue line) and $5 \mathrm{~Hz}$ (red line) with various temporal offsets $\left(\Delta \mathrm{t}=\mathrm{t}_{\text {post }} \mathrm{t}_{\mathrm{pre}}\right), \tau_{\mathrm{bAP}, \mathrm{s}}=25 \mathrm{~ms}$ and $\tau_{\mathrm{NMDA}, \mathrm{s}}=152 \mathrm{~ms}$. (b) Maximum peak NMDAr-[Ca $\left.{ }^{2+}\right]$ generated by $30-100$ triplet pairings delivered at $0.5 \mathrm{~Hz}$ (blue line) and $5 \mathrm{~Hz}$ (red line) with the same parameter values as (a). Horizontal dashed lines represent putative thresholds for the induction of LTP and LTD ( $\theta_{\text {LTP }}$ and $\theta_{\text {LTD }}$ respectively) which are subsequently used to inform parameterisation of the thresholded Hill functions that control kinase and phosphotase activity respectively. Vertical dashed line represents $\Delta \mathrm{t}=0 \mathrm{~ms}$. (c) Total change in synaptic weight generated by 100 spike pairings delivered at $5 \mathrm{~Hz}$ with $\tau_{\mathrm{bAP}, \mathrm{s}}=25 \mathrm{~ms}$, $\tau_{\mathrm{NMDA}, s}=152 \mathrm{~ms}, \beta_{\mathrm{P}}=0.35$ and $\beta_{\mathrm{D}}=0.175$. (d) Total change in synaptic weight generated by 100 triplet pairings delivered at $5 \mathrm{~Hz}$ with the same parameter values as (c). (e) Total change in synaptic weight generated by 100 triplet pairings delivered at $0.5 \mathrm{~Hz}$ with the same parameter values as (c). (f) Total change in synaptic weight generated by 30 triplet pairings delivered at $5 \mathrm{~Hz}$ with the same parameter values as (c). Horizontal dashed line represents zero change in total synaptic weight, and vertical dashed line represents $\Delta \mathrm{t}=0 \mathrm{~ms}$ 

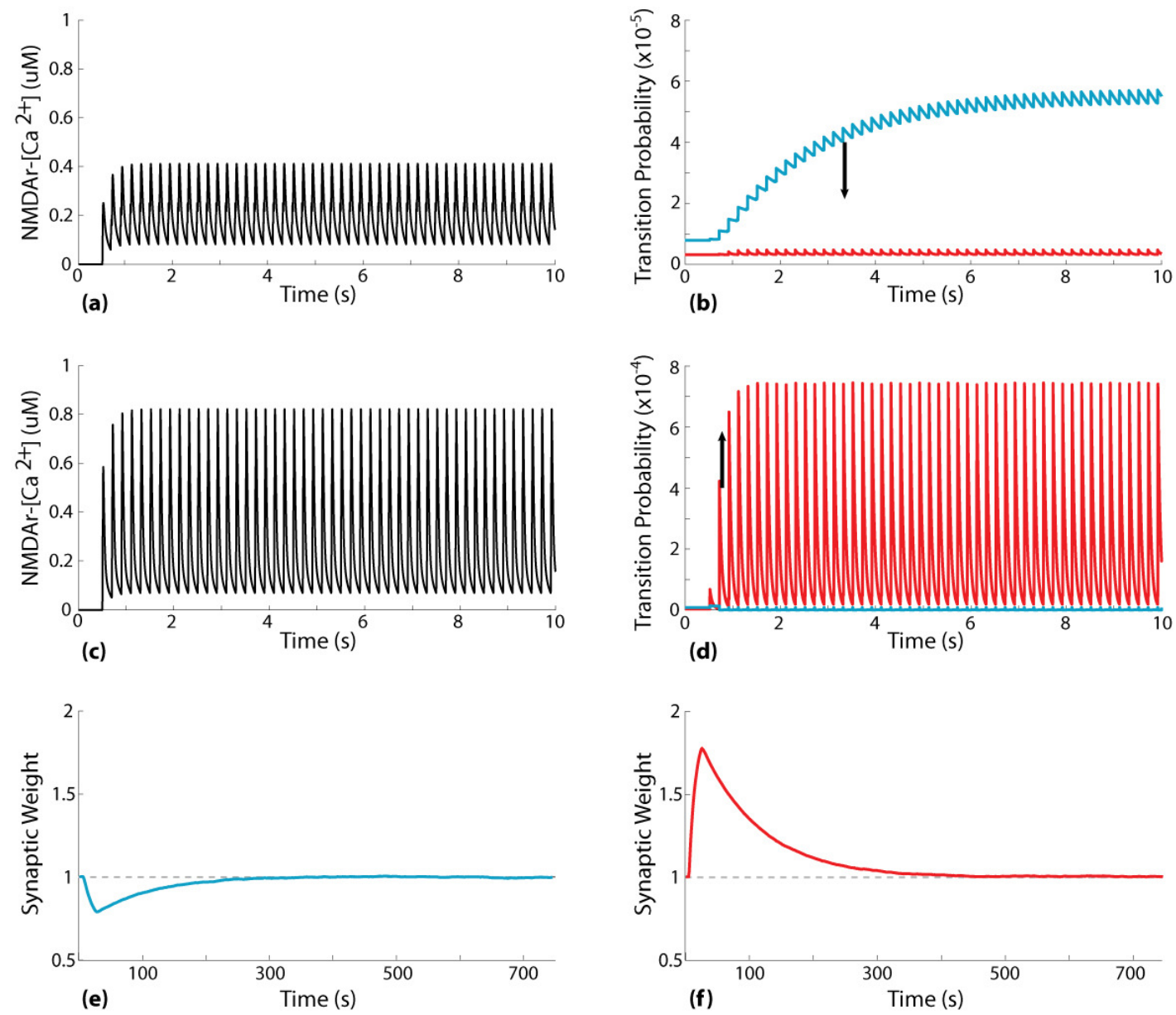

Fig. 3 Local dynamics of intracellular Calcium, kinase and phosphotase activity and synaptic weight in the dendritic spine during potentiating and depressing causal and acausal spike pairings. (a) NMDAr- $\left[\mathrm{Ca}^{2+}\right]$ dynamics in the dendritic spine during a typical depressing stimulus $-5 \mathrm{~Hz}$ triplet pairing with $\Delta \mathrm{t}=\mathrm{t}_{\text {post }} \mathrm{t}_{\text {pre }}=-15 \mathrm{~ms}, \tau_{\mathrm{bAP}, \mathrm{s}}=25 \mathrm{~ms}$ and $\tau_{\mathrm{NMDA}, \mathrm{s}}=152 \mathrm{~ms}$. (b) $\mathrm{NMDAr}-\left[\mathrm{Ca}{ }^{2+}\right]$ dynamics in the dendritic spine during a typical potentiating stimulus $-5 \mathrm{~Hz}$ triplet pairing with with $\Delta \mathrm{t}=\mathrm{t}_{\text {post }} \mathrm{t}_{\text {pre }}=15 \mathrm{~ms}$ and the same parameter values as (a). (c) Kinase (red) and phosphotase (blue) dynamics during a typical depressing stimulus with the same parameter values as $(\mathrm{a}), \beta_{\mathrm{P}}=0.35$ and $\beta_{\mathrm{D}}=0.175$. The black arrow indicates the time at which the first synaptic weight makes the transition from a low to high weight state. (d) Kinase (red) and phosphotase (blue) dynamics during a typical potentiating stimulus with the same parameter values as (b),$\beta_{\mathrm{P}}=0.35$ and $\beta_{\mathrm{D}}=0.175$.. The black arrow indicates the time at which the first synaptic weight made the transition from a high to low weight state. (e) Decay of LTD and (f) LTP following the potentiating and depressing stimulation protocols illustrated in (a-d)

\subsection{The Range of Temporal Interactions, Slow Time Constants and Skew}

The temporal extent of spike pair interactions at low frequencies is primarily determined by the slow time constant of the bAP, which dictates the duration of residual depolarisation and therefore partial relief of NMDA blockade in the spine following a post-synaptic action potential; and that of the NMDA receptor, which dictates the duration of glutamate binding following pre-synaptic input [18]. In the simulations described above, the slow time constant of the NMDA receptor $\left(\tau_{\mathrm{NMDA}, \mathrm{s}}=152 \mathrm{~ms}\right)$ is much larger than that of the bAP $\left(\tau_{\mathrm{bAP}, \mathrm{s}}=25 \mathrm{~ms}\right)$, and hence pre-post interactions extend over a larger temporal window than post-pre interactions. This accounts for the induction of depression over a greater range of positive than negative temporal offsets with spike pairing stimulation (Figure 2c); and for the induction of more significant depression around $\Delta \mathrm{t}=-100 \mathrm{~ms}$ than $\Delta \mathrm{t}=-50 \mathrm{~ms}$ in triplet pairing simulations (Figure 2d). In the latter case, post-synaptic bursts at large negative temporal offsets interact more significantly with decaying NMDA receptor activation from the previous pre-synaptic input. This appears to be quantitatively at odds with empirical data, which suggests that smaller net weight change should be generated as $\Delta \mathrm{t} \rightarrow \pm 100 \mathrm{~ms}$ at $5 \mathrm{~Hz}$ (see Figure 1), and thus that consecutive triplet pairings should not interact at this stimulation frequency.

Adjusting the relative magnitude of these slow time constants independently modulates the positive and negative temporal extent of NDMAr- $\left[\mathrm{Ca}^{2+}\right]$ influx generated by spike or triplet pairings in this model, and thus the skew of peak NMDAr- $\left[\mathrm{Ca}^{2+}\right]$ values and the resultant learning curve (Figure $4 \mathrm{a}-\mathrm{d}$ ). In 
order to produce Gaussian plasticity profiles, these parameters must be manipulated to provide an approximately Gaussian distribution of peak NMDA- $\left[\mathrm{Ca}^{2+}\right]$ values from spike-timing stimulation protocols. However, it may be an oversimplification to use a Gaussian fit to experimental data, as statistical tests demonstrate that the learning curves described in the acute slice by Wittenberg and Wang [29] exhibit negative skew $(\gamma=-0.39$ for the depression only learning rule illustrated in Figure 1c, for example).
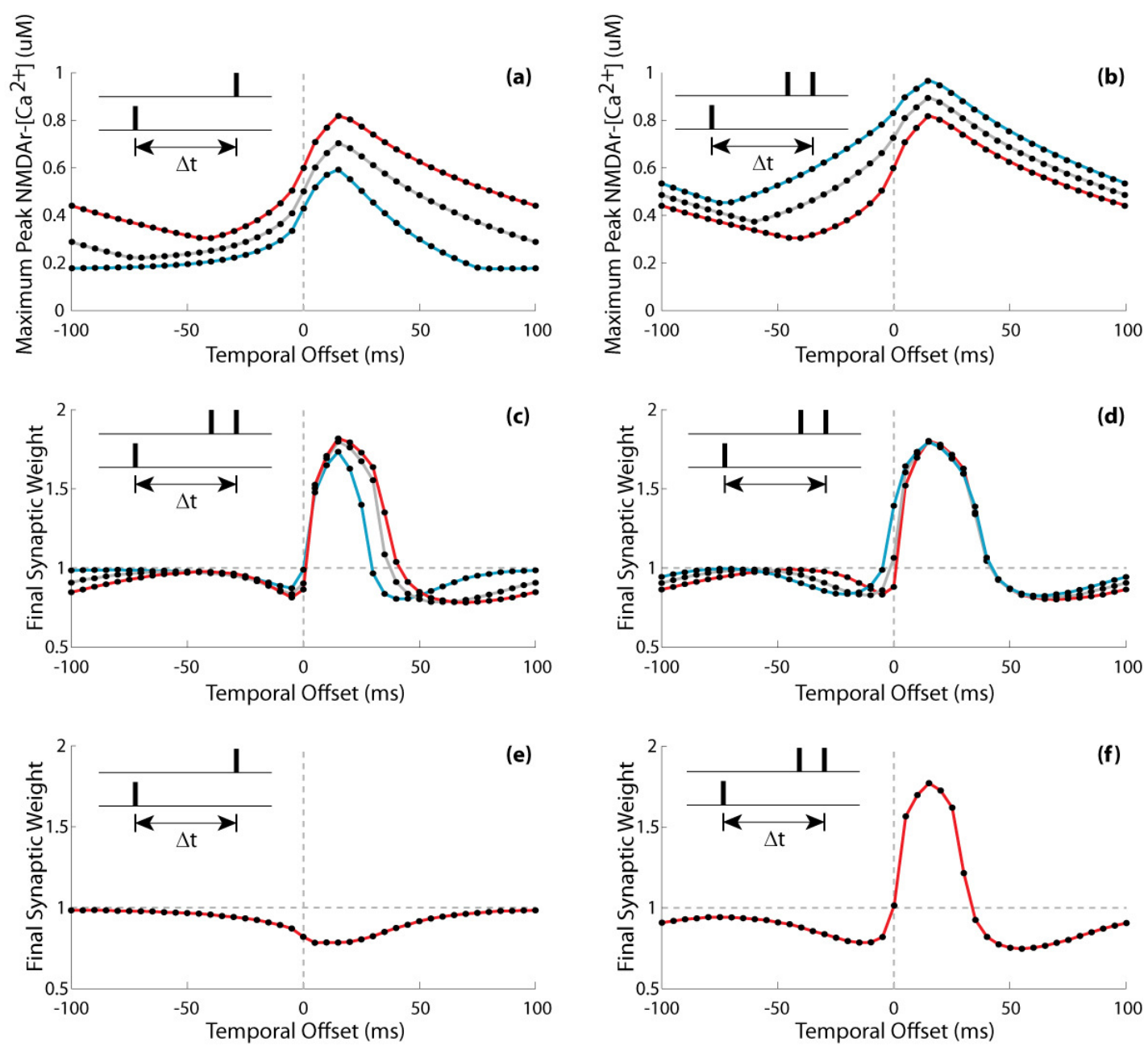

Fig. 4 Effects of adjusting NMDAr and bAP time constants on peak NMDAr-[Ca $\left.{ }^{2+}\right]$ and synaptic weight change. (a) Peak NMDAr- $\left[\mathrm{Ca}^{2+}\right]$ values generated by 100 triplet pairings delivered at $5 \mathrm{~Hz}$ and various temporal offsets $\left(\Delta t=t_{\text {post }}-t_{\text {pre }}\right)$ with $\tau$. $\mathrm{bAP}, \mathrm{s}=25 \mathrm{~ms}$ and $\tau_{\mathrm{NMDA}, \mathrm{s}}=50 \mathrm{~ms}$ (blue line); $\tau_{\mathrm{NMDA}, \mathrm{s}}=100 \mathrm{~ms}$ (grey line); or $\tau_{\mathrm{NMDA}, \mathrm{s}}=152 \mathrm{~ms}$ (red line). (b) Peak NMDAr-[Ca $\left.{ }^{2+}\right]$ values generated by 100 triplet pairings delivered at $5 \mathrm{~Hz}$ with $\tau_{\mathrm{NMDA}, \mathrm{s}}=152 \mathrm{~ms}$ and $\tau_{\mathrm{bAP}, \mathrm{s}}=25 \mathrm{~ms}$, (red line); $\tau_{\mathrm{bAP}, \mathrm{s}}=55 \mathrm{~ms}$ (grey line); or $\tau_{\text {. }}$ ${ }_{\mathrm{bAP}, \mathrm{s}}=85 \mathrm{~ms}$ (blue line). (c) Synaptic weight change generated by 100 triplet pairings delivered at $5 \mathrm{~Hz}$ with $\tau_{\mathrm{bAP}, \mathrm{s}}=25 \mathrm{~ms}$ and $\tau_{\mathrm{NMDA}, \mathrm{s}}=50 \mathrm{~ms}, \beta_{\mathrm{P}}=0.175$ and $\beta_{\mathrm{D}}=0.04$ (blue line); $\tau_{\mathrm{NMDA}, \mathrm{s}}=100 \mathrm{~ms}, \beta_{\mathrm{P}}=0.25$ and $\beta_{\mathrm{D}}=0.09$ (grey line); or $\tau_{\mathrm{NMDA}, \mathrm{s}}=152 \mathrm{~ms}, \beta_{\mathrm{P}}=0.35$ and $\beta_{\mathrm{D}}=0.175$ (red line). (d) Synaptic weight change generated by 100 triplet pairings delivered at $5 \mathrm{~Hz}$ with $\tau_{\mathrm{NMDA}, \mathrm{s}}=152 \mathrm{~ms}, \tau$. ${ }_{\mathrm{bAP}, \mathrm{s}}=25 \mathrm{~ms}, \beta_{\mathrm{P}}=0.35$ and $\beta_{\mathrm{D}}=0.175$ (red line); $\tau_{\mathrm{bAP}, \mathrm{s}}=55 \mathrm{~ms}, \beta_{\mathrm{P}}=0.425$ and $\beta_{\mathrm{D}}=0.25$ (grey line); or $\tau_{\mathrm{bAP}, \mathrm{s}}=85 \mathrm{~ms}, \beta_{\mathrm{P}}=0.5$ and $\beta_{\mathrm{D}}=0.33$ (blue line). (e) Synaptic weight change generated by 100 spike pairings delivered at $5 \mathrm{~Hz}$ with $\tau_{\mathrm{bAP}, \mathrm{s}}=55 \mathrm{~ms}, \tau_{\mathrm{NMDA}, \mathrm{s}}=100 \mathrm{~ms}$, $\beta_{\mathrm{P}}=0.3$ and $\beta_{\mathrm{D}}=0.125$. (f) Synaptic weight change generated by 100 triplet pairings delivered at $5 \mathrm{~Hz}$ with the same parameter values as (e)

Hence, in order to account for the induction of depression at $\Delta \mathrm{t}<-50 \mathrm{~ms}$; to avoid spurious pre-post pairings at $\Delta \mathrm{t}>\sim 80 \mathrm{~ms}$; and to increase the negative skew of the NMDAr- $\left[\mathrm{Ca}^{2+}\right]$ profile and resultant learning curves, we repeat the simulations described above with $\tau_{\mathrm{bAP}, \mathrm{s}}=55 \mathrm{~ms}$ and $\tau_{\mathrm{NMDA}, \mathrm{s}}=100 \mathrm{~ms}$ (Figure $4 \mathrm{e}, \mathrm{f}$ ). This represents a significantly longer time constant for the bAP than that employed in previous modelling studies; although there is little empirical data available to guide the choice of this parameter value [18, 48, 54-56]. The slow time constant of Calcium influx through the NMDA receptor is also shorter than that measured experimentally, which has been shown to correspond well with the time course of deactivation following glutamate release [ 150ms; 57]. However, that study measured the time course of Calcium influx following unitary NMDAr activation, whereas we are concerned 
with NMDAr kinetics when significant post-synaptic depolarisation follows glutamate binding by $\sim 50$ $100 \mathrm{~ms}$, which may be significantly more complex [46].

\subsection{Induction of Synaptic Plasticity by Other Stimulation Protocols}

Having constrained the parameters of the plasticity model to account for recent observations of triphasic hippocampal STDP, we now examine whether it is also possible to replicate synaptic plasticity data obtained using other stimulation protocols in order to provide a unified account of weight change dictated by NMDAr- $\left[\mathrm{Ca}^{2+}\right]$. Firstly, we examine tetanic stimulation - whereby a set number of pre-synaptic inputs are applied periodically at a constant firing rate. This form of stimulation typically generates a BCM-type learning curve that is well replicated by this model (Figure 5a, b); composed of competitive and co-activated potentiation and depression only learning curves that are revealed under selective pharmacological blockade of phosphotase or kinase activity respectively (Figure 5c, d). In both cases, however, the firing rate at which LTD and LTP are first expressed in the model is significantly higher than that observed experimentally [38].
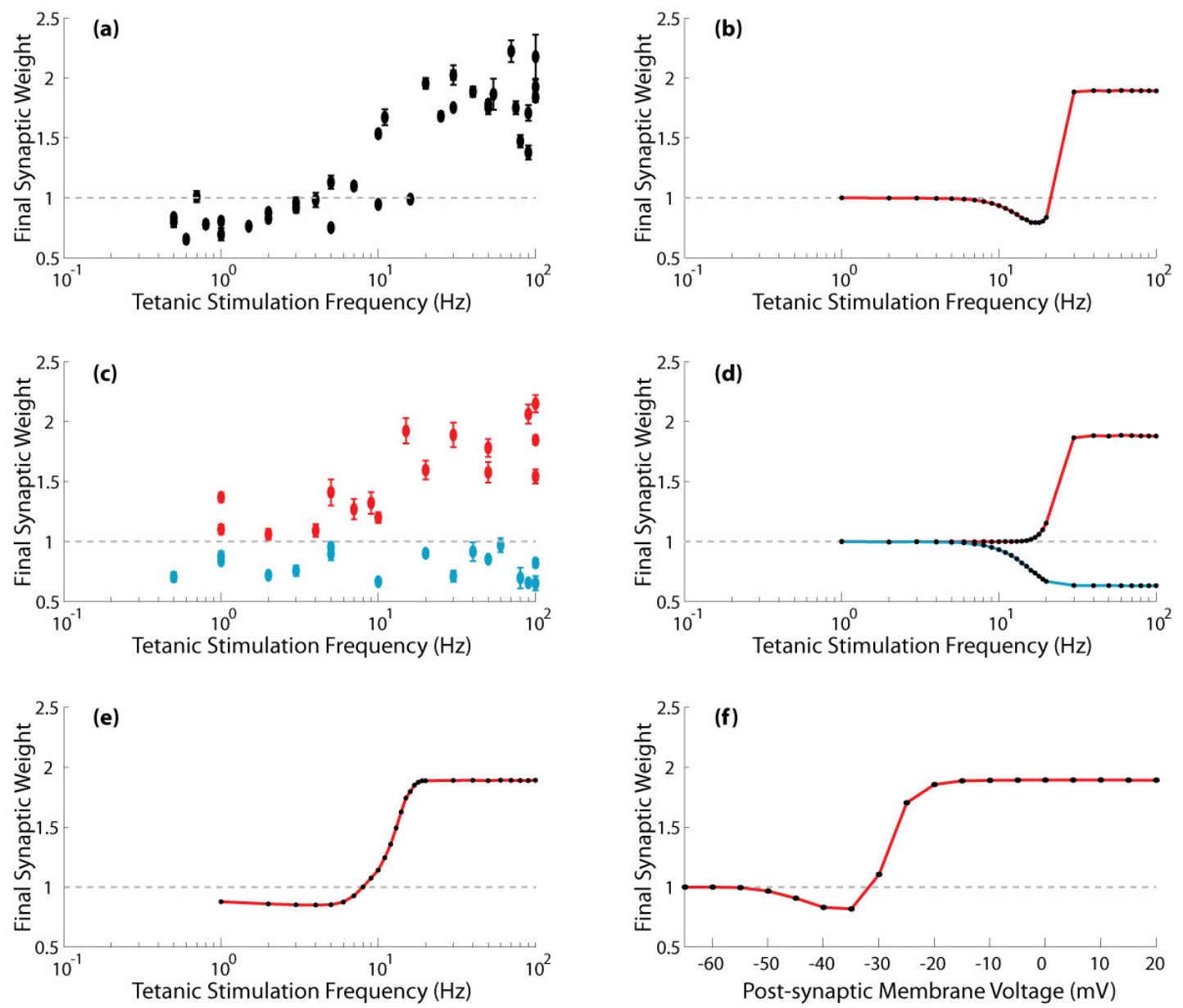

Fig. 5 Synaptic plasticity induced by tetanic stimulation, post-synaptic depolarisation and under selective pharmacological blockade. (a) Experimentally observed synaptic weight change induced by 900 pulses of periodic pre-synaptic stimulation delivered at various firing rates from O'Connor, Wittenberg and Wang [36]. (b) Overall synaptic weight change generated in this model when the same form of stimulation is applied with $\tau_{\mathrm{bAP}, \mathrm{s}}=55 \mathrm{~ms}, \tau_{\mathrm{NMDA}, \mathrm{s}}=100 \mathrm{~ms}, \beta_{\mathrm{P}}=0.3$ and $\beta_{\mathrm{D}}=0.125$. (c) Experimentally observed weight change induced by 900 pulses of pre-synaptic stimulation delivered at various firing rates in the presence of selective pharmacological blockade of kinase (blue) or phosphotase (red) activity from O'Connor, Wittenberg and Wang [36]. (d) Overall synaptic weight change generated in our model when the same form of stimulation is applied under conditions of putative kinase (blue) or phosphotase (red) blockade (i.e. $\mathrm{k}_{\mathrm{P}}=0$ or $\mathrm{k}_{\mathrm{D}}=0$, see Methods) with all other parameter values the same as in (b). (e) Overall synaptic weight change generated in our model by 900 pulses of periodic pre-synaptic stimulation delivered at various firing rates with stochastic post-synaptic activity that follows the statistics described by Wittenberg and Wang [29] and the same parameter values as (b). (f) Overall synaptic weight change generated in our model by 100 pre-synaptic inputs delivered at $2 \mathrm{~Hz}$ while the post-synaptic membrane voltage is held fixed at various levels of depolarisation, with the same parameter values as (b)

One issue with the interpretation of empirical plasticity data obtained using tetanic stimulation protocols is the fact that post-synaptic activity is rarely recorded, but has a significant impact on the 
magnitude of NMDAr- $\left[\mathrm{Ca}^{2+}\right]$ generated. In fact, experimental evidence suggests that post-synaptic activity is a necessary requirement for any form of synaptic plasticity, although contradictory reports do exist [32, 58, 59]. Wittenberg and Wang [29] provide unique data regarding the induction of LTD by a single LFS protocol ( 900 pulses at $3.3 \mathrm{~Hz}$ ), in that the response of the post-synaptic neuron was recorded throughout pre-synaptic stimulation - as 200 action potentials which each followed input volleys with a latency of $6.2 \pm 4 \mathrm{~ms}$. Such causal spike pairings might be expected to generate LTP by a conventional STDP rule, highlighting a critical issue with previous phenomenological models [32]. When we repeat the simulations illustrated in Figure $5 \mathrm{~b}$ in the presence of stochastic post-synaptic activity with the same statistics the BCM-type learning rule is retained, and the threshold firing rate for expression of both LTD and LTP is reduced to more realistic levels (Figure 5e).

Next, we examine the changes in synaptic weight generated when pre-synaptic stimulation is paired with different levels of post-synaptic depolarisation (Figure 5f) or theta burst stimulation (TBS) is applied (see Methods). In each case, this model qualitatively replicates experimental data and the results of previous modelling studies - both solely pre-synaptic TBS and simultaneous pre- and postsynaptic TBS generating saturated LTP with a final synaptic weight of $1.89 \pm 0.01$ [48, 60-62].

\subsection{Effects of bAP Attenuation, Stimulation Frequency and Decay of Early-phase Plasticity}

Having established that this plasticity model can account for a wide range of experimental data, we can now make experimental predictions regarding the degree and direction of synaptic weight change induced by set activity patterns when various experimental parameters are manipulated. For example, in the simulations described above, the amplitude of the bAP is a critical parameter which determines the level of depolarisation - and therefore voltage-dependent relief of NMDAr - following a postsynaptic action potential. It is well known that bAP amplitude attenuates significantly with distance from the soma, primarily due to the activity of A-type $\mathrm{K}^{+}$channels, and this may have a critical impact on the nature of synaptic weight change generated by identical stimulation protocols at proximal and distal dendrites $[56,63,64]$. Simulations indicate that a reduction in the value of $\mathrm{V}_{\mathrm{bp} \text {,max }}$ generates a reduction in the magnitude of LTP and the temporal extent of both LTP and LTD generated by STDP protocols (Figure 6a). This matches recent experimental data which examined the synaptic weight change incurred by identical spike-timing stimulation protocols at proximal and distal inputs to cortical pyramidal neurons - although similar results from CA1 pyramidal neurons are so far lacking [65].

We can also examine the effect of adjusting the frequency of spike and triplet pairing stimulation on synaptic weight change. This form of activity approximates that observed in the hippocampus during stereotyped learning behaviour and spatial exploration - when active pyramidal neurons fire single spikes or complex bursts at approximately theta frequency. In these simulations, spike pairings generate a broad depression only curve at low frequencies $(<7 \mathrm{~Hz})$, and an increasing magnitude and temporal extent of potentiation and depression centred around short, positive temporal offsets at higher frequencies $(\geq 8 \mathrm{~Hz}$; Figure $6 \mathrm{~b}$ ). Similarly, triplet pairings generate a broad depression only curve reminiscent of that generated by spike pairings at low frequencies $(<4 \mathrm{~Hz})$, and an increased temporal extent of potentiation and magnitude of depression at higher frequencies $(\geq 5 \mathrm{~Hz}$; Figure $6 \mathrm{c}$ ). The requirement for both moderate stimulation frequency and post-synaptic bursting for LTP, in contrast to low stimulation frequency and / or single post-synaptic spikes for LTD, dictates that bi-directional synaptic plasticity is induced over a much wider range of conditions than that observed in previous modelling studies [18].

Finally, it is interesting to note that the modified Hill functions utilised here allow the NMDAr-[Ca $\left.{ }^{2+}\right]$ threshold at which potentiation and depression are expressed to be modulated in a straightforward manner by adjusting the corresponding value of $\beta$ (see Methods). Hence, a process of metaplasticity that is, an activity dependent change in the level of stimulation required to induce LTP or LTD - can easily be incorporated into the model. Figure $6 \mathrm{~d}$ illustrates the learning curves generated by $5 \mathrm{~Hz}$ triplet pairing when the thresholds for kinase and phosphotase are increased, limiting their expression and effectively generating depression or potentiation only learning rules. 

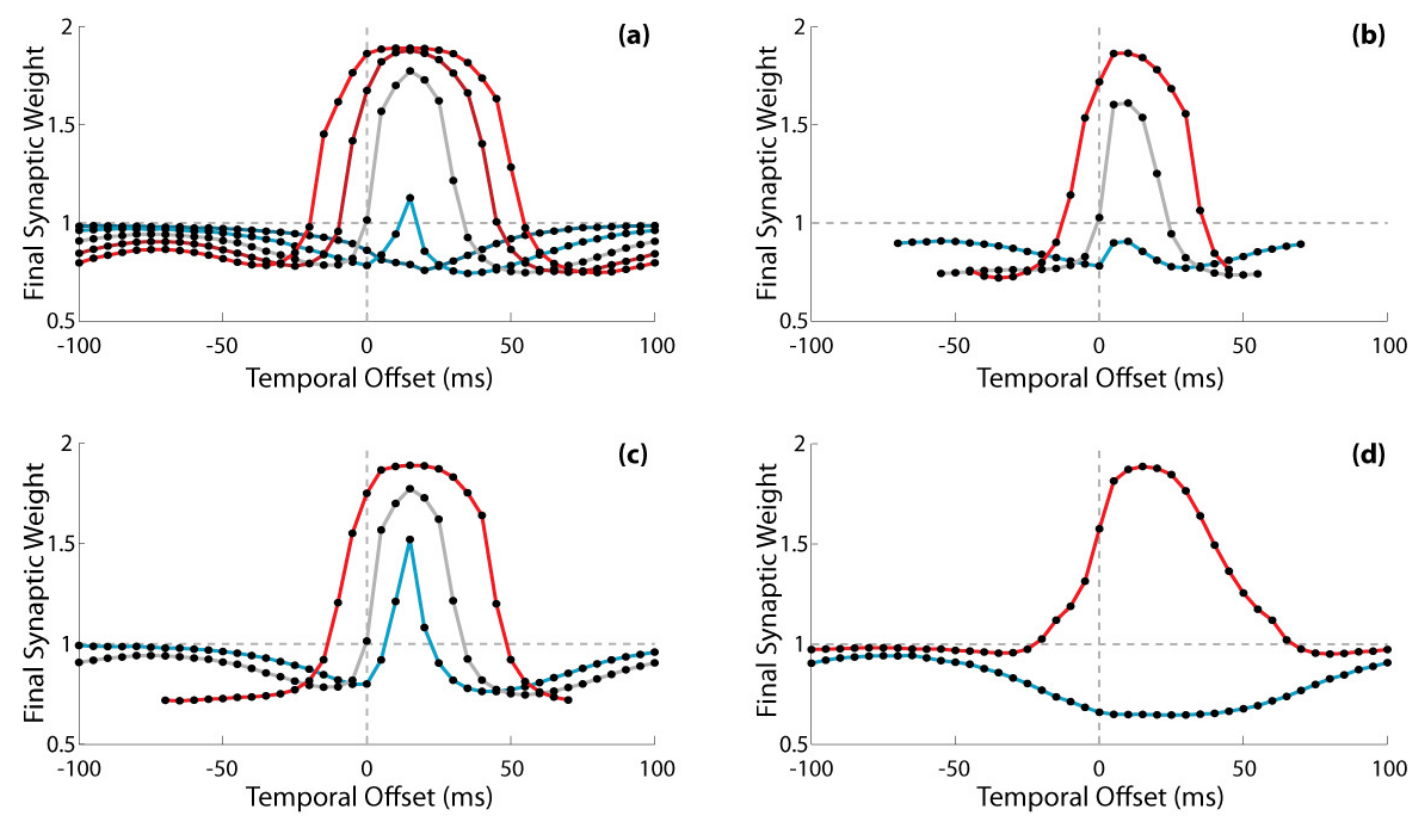

Fig. 6 Effects of adjusting bAP amplitude $\left(\mathrm{V}_{\mathrm{bp}, \max }\right)$, stimulation frequency, kinase and phosphotase activation thresholds on synaptic weight change. (a) Synaptic weight change generated by 100 triplet pairings delivered at $5 \mathrm{~Hz}$ with various temporal offsets $\left(\Delta \mathrm{t}=\mathrm{t}_{\mathrm{post}} \mathrm{t}_{\mathrm{pre}}\right), \tau_{\mathrm{bAP}, \mathrm{s}}=55 \mathrm{~ms}, \tau_{\mathrm{NMDA}, \mathrm{s}}=100 \mathrm{~ms}, \beta_{\mathrm{p}}=0.3, \beta_{\mathrm{D}}=0.125$ and $\mathrm{V}_{\mathrm{bp}, \max }=33 \mathrm{mV}$ (blue line), $\mathrm{V}_{\mathrm{bp}, \max }=50 \mathrm{mV}, \mathrm{V}_{\mathrm{bp}}$, ${ }_{\max }=67 \mathrm{mV}$ (grey line), $\mathrm{V}_{\mathrm{bp}, \max }=83 \mathrm{mV}$ and $\mathrm{V}_{\mathrm{bp}, \max }=100 \mathrm{mV}$ (red line). (b) Synaptic weight change generated by 100 spike pairings delivered at $7 \mathrm{~Hz}$ (blue line), $9 \mathrm{~Hz}$ (grey line), and $11 \mathrm{~Hz}$ (red line) with the same parameter values as (a). (c) Synaptic weight change generated by 100 triplet pairings delivered at $3 \mathrm{~Hz}$ (blue line), $5 \mathrm{~Hz}$ (grey line), and $7 \mathrm{~Hz}$ (red line) with the same parameter values as (a) (d) Synaptic weight change generated by 100 triplet pairings delivered at $5 \mathrm{~Hz}$ with $\beta_{\mathrm{p}}=0.5, \beta_{\mathrm{D}}=0.125$ (blue line) and $\beta_{\mathrm{P}}=0.3, \beta_{\mathrm{D}}=0.175$ (red line)

\section{Discussion}

Recent empirical examinations of spike-timing dependent plasticity at the CA3-CA1 synapse have demonstrated that changes in synaptic conductance are jointly dependent on the temporal offset of preand post- synaptic firing, number of post-synaptic spikes fired, frequency of spike or triplet pairings and duration of stimulation [28-30]. Here, we provide the first demonstration that the Calcium control hypothesis, which purports that the dynamics of NMDAr-dependent Calcium influx into dendritic spines is sufficient to account for the magnitude and direction of synaptic weight changes induced by different stimulation protocols, can qualitatively account for this joint dependency. As with the original formulation of the Calcium control hypothesis, our motivation was to reproduce a wide range of plasticity data using a minimal number of assumptions and thereby provide a parsimonious model that is suitable for network level simulations $[18,47]$. In addition to those made previously, we have incorporated co-activated and competitive kinase and phosphotase pathways with distinct time constants that dictate probabilistic transitions between binary synaptic states to provide a unified computational model of hippocampal synaptic plasticity - a process that is critically implicated in mammalian declarative memory function. Importantly, this formulation relies on an explicit consideration of the dynamics of NMDAr- $\left[\mathrm{Ca}^{2+}\right]$ and bistable synaptic weights, as opposed to a summation of peak or integrated Calcium influx and continuous synaptic weights [45].

The dynamics of kinase and phosphotase in this model are controlled by modified Hill functions inspired by recent advances in systems biology [49, 50]. These were developed to provide a quantitative kinetic description of enzymes and transcription factors that are sequestered by inhibitors or antagonists into inactive complexes below some threshold or equivalence point. This sequestration mechanism, which is widespread in genetic and regulatory networks, has several properties that make it particularly useful for modelling synaptic plasticity processes, providing a threshold for activation below which the accumulation of active regulatory molecules is effectively buffered and above which an ultrasensitive response that approaches bistability is generated. It is also straightforward to parameterise the modified Hill functions to suit the demands of any particular synaptic plasticity simulation, by matching the threshold value $\beta$ to the peak NMDAr- $\left[\mathrm{Ca}^{2+}\right]$ values generated by different forms of stimulation at which the expression of potentiation or depression is required. 
A number of predictions made by the synaptic plasticity model presented here might be experimentally verified in order to provide further support for our approach and highlight areas where revisions are required. For example, we suggest that the induction of LTD by post-pre spike pairings over a range of $-60 \mathrm{~ms}<\Delta \mathrm{t}<0 \mathrm{~ms}$ implies that the slow after-depolarisation generated in dendritic spines by a bAP should follow a similar time course. It is very difficult to test this conjecture experimentally. It has also been suggested that one of several alternative mechanisms may serve as the second coincidence detector required for the induction of LTD by spike and triplet pairing stimulation protocols [42, 45, 66]. By elaborating the secondary messenger pathways in the plasticity model to more closely replicate the activity of CaM, CaMKII, PP1, PP2A, I1 and other proteins implicated in plasticity expression, as well as additional sources of internal and external Calcium influx, it may be possible to more accurately appraise the contributions made by bAPs to synaptic plasticity [7, 46, 67]. However, the complexity of such models and associated computational cost might also render them unsuitable for network level simulation.

The results presented here also suggest that, in order to avoid spurious pre-post pairings at $\Delta \mathrm{t}>\sim 60 \mathrm{~ms}$, the NMDA receptor should not allow significant Calcium influx when depolarisation follows glutamate binding by a similar temporal offset. This prediction could be tested through experimental measurements of the NMDAr- $\left[\mathrm{Ca}^{2+}\right]$ current generated by spike and triplet pairing protocols at different stimulation frequencies. It is well known that the activity-dependent NR2A / NR2B subunit composition of NMDAr has a significant influence on the properties and temporal profile of synaptic currents, particularly at the high and low firing rates associated with LTP and LTD induction [68, 69]. Measuring the changes in synaptic strength generated by spike and triplet pairing protocols under regimes of selective pharmacological blockade would also allow the dynamics of kinase and phosphotase activity to be more precisely delineated, providing further data to constrain the corresponding kinetic models. Similarly, recording post-synaptic activity over a range of tetanic stimulation frequencies would allow the specific profile of NMDAr-dependent Calcium influx generated to be more accurately replicated in simulation.

The dependence of both LTP and LTD on stimulation frequency suggests that more significant plasticity will be generated by a set pattern of synaptic activity in the hippocampus when theta frequency increases. Although some empirical studies have reported a correlation between hippocampal theta frequency during encoding and subsequent memory performance in humans and other mammals, it has also been demonstrated that environmental novelty - which is associated with enhanced learning and plasticity - causes a decrease in CA1 theta frequency and might therefore produce less significant changes in synaptic conductance [70-73]. However, it seems likely that multiple alterations in neural activity or neuromodulatory tone are generated by novelty or enhanced learning and may have a more significant effect on the dynamics of synaptic plasticity. The requirement for multiple post-synaptic spikes to induce LTP, for example, implies that bursting may be the primary mode of encoding salient information in the hippocampus [74, 75]. Further empirical studies are required to delineate the changes in neural activity observed during periods of enhanced learning, and the manner in which these changes contribute to the induction of bidirectional synaptic plasticity.

The model presented here also exhibits several limitations that might be addressed by future theoretical studies. For example, the magnitude of weight change induced by different stimulation protocols in this model is generally smaller than that observed empirically, despite the fact that the relative frequency and conductance of potentiated and depressed synapses are matched to published data [36, 37, 44; see Methods]. Furthermore, specific AMPAr and NMDAr conductances are not affected by changes in putative synaptic weight which, although in line with many theoretical studies of plasticity induction, is clearly at odds with the situation in vivo [18, 42-48]. It has been demonstrated that changes in AMPAr conductance associated with the expression of long-term synaptic plasticity are accompanied by concomitant changes in NMDAr conductance [69, 76]. This has interesting implications for further synaptic plasticity - as both the level of depolarisation in the spine and the level of NMDAr-dependent Calcium influx generated by that depolarisation will be concurrently modulated, possibly contributing to the 'lock-in' of changes in synaptic conductance observed experimentally [37].

It is also important to note that the results presented here are only intended to model long-term plasticity of CA3-CA1 synapses in the hippocampus. Experimental studies have demonstrated that the Calcium control hypothesis does not hold true for excitatory synapses between cortical pyramidal cells - at which there is no evidence for the existence of a triphasic STDP rule. A variety of alternative 
mechanisms, including metabotropic glutamate receptor, pre-synaptic NMDAr and retrograde endocannabinoid signalling, have been implicated in the induction of LTD by spike-timing stimulation protocols in cortex [77-80]. It seems likely that this dichotomy in synaptic plasticity mechanisms between cortex and hippocampus might both reflect and be reflected by functional differences in neural processing mediated by these regions.

We have demonstrated that this plasticity model can account for the decay of early-phase plasticity over a period of $\sim 1$ hour following changes in synaptic conductance, facilitating the incorporation of plasticity-related-protein (PRP) production, synaptic tagging and capture (STC) processes in order to examine hetero-synaptic interactions during more realistic long-term activity patterns [53]. It is also well known that CA1 pyramidal neurons receive predominantly inhibitory input from multiple sources during stereotyped learning and spatial exploration, and that changes in the level of inhibition during development significantly modulate the degree and direction of synaptic plasticity incurred by spiketiming stimulation protocols [34]. Hence, it seems prescient to extend this model to include realistic patterns of inhibitory input, in order to provide a more comprehensive appraisal of synaptic plasticity at the CA3-CA1 synapse. The distribution of inhibitory and excitatory inputs with different properties at different locations across the dendritic tree will generate more complex patterns of post-synaptic depolarisation and Calcium influx, possibly allowing useful nonlinear computations to be performed. Recent studies have described several forms of heterosynaptic plasticity in pyramidal neurons that might also be explained by the Calcium control hypothesis, allowing the nature of active dendritic processing to be more precisely elucidated [81-84].

Finally, it is well established that processes of synaptic plasticity are accompanied by concurrent and interacting processes of homeostatic and intrinsic plasticity which regulate neural activity over longer timescales $[12,85]$. Recent computational modelling suggests that the interaction of these processes is critical to establish and maintain appropriate conditions for transient dynamics during cognitive processing, and the examination of a unified model of neural and synaptic plasticity is therefore a critical direction for future theoretical studies [86-89]. More generally, an examination of the synaptic and neural dynamics generated by the triphasic STDP rule in network models of hippocampal function with more realistic activity patterns, including theta modulation and phase precession, would contribute significantly to the understanding of hippocampal function during putative learning behaviour [90].

\section{Methods}

\subsection{Neuron Model}

The vast majority of afferent excitatory synapses on cortical pyramidal neurons are found on dendritic spines - small, membranous protrusions that are connected to the dendritic tree by a narrow neck [91]. Due to the limited diffusion of biochemical signals including Calcium through this neck, dendritic spines effectively compartmentalise synaptic processes and thereby promote their independence [57]. In this model, we examine $N$ independent, passive dendritic spines with surface area $\mathrm{A}_{\text {spine }}$ whose membrane potential $\mathrm{V}_{\mathrm{N}}$ is dictated solely by leak conductance according to Eq. 1; where $\mathrm{C}_{\mathrm{m}}$ is the membrane capacitance, $\mathrm{I}_{\mathrm{N}}$ is the total synaptic current to the Nth spine, $\mathrm{g}_{\mathrm{L}}$ is the (maximum) leak conductance and $\mathrm{E}_{\mathrm{L}}$ is the reversal potential of the leak current.

$$
\frac{d V_{N}}{d t}=\frac{1}{C_{m}}\left(\frac{I_{N}}{A_{\text {spine }}}-\bar{g}_{L}\left(V_{N}-E_{L}\right)\right)
$$

Each spine has AMPA and NMDA mediated synaptic currents with kinetics based on experimental recordings and governed by Eq. 2 [92, 93]. AMPAr conductances are modelled as a single exponential with instantaneous rise time and subsequent decay with a time constant of $\tau_{\mathrm{AMPA}}=5.26 \mathrm{~ms}$. NMDAr conductances are modelled as the sum of fast and slow exponentials with time constants $\tau_{\mathrm{NMDA}, \mathrm{f}}=$ $1.485 \mathrm{~ms}$ and $\tau_{\mathrm{NMDA}, \mathrm{s}}$ respectively. The term $\mathrm{G}_{\mathrm{NMDA}}$ describes the additional voltage dependence due to the blockade of NMDA receptors by $\mathrm{Mg}^{2+}$ [94], while $\mathrm{B}_{\mathrm{NMDA}}$ is a normalisation factor which ensures that the peak conductance is equal to the maximum conductance. 


$$
\begin{aligned}
& I_{N}=I_{A M P A}+I_{N M D A} \\
& I_{A M P A}=-g_{A M P A} P_{A M P A}\left(V_{N}-E_{A M P A}\right) \\
& P_{A M P A}(t)=\exp ^{\left(t_{0}-t\right) / \tau_{A M P A}} \\
& I_{N M D A}=-g_{N M D A} P_{N M D A} G_{N M D A}\left(V_{N}-E_{N M D A}\right) \\
& P_{N M D A}(t)=B_{N M D A}\left(\exp ^{\left(t_{0}-t\right) / \tau_{N M D A_{s}}}-\exp ^{\left(t_{0}-t\right) / \tau_{N M D A_{f}}}\right)
\end{aligned}
$$

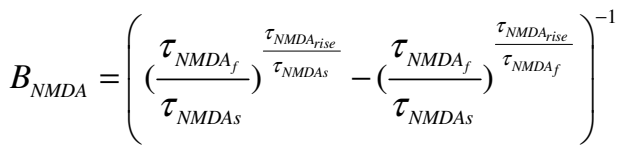

$$
\begin{aligned}
& G_{N M D A}=\frac{1}{\left(1+\frac{\left[M g^{2+}\right]}{3.57} \exp ^{-V_{N} / 16.13}\right)}
\end{aligned}
$$

Another significant source of depolarisation in the dendritic spine is provided by backpropagating action potentials (bAPs) from the soma $[54,55,95]$. In accordance with previous modelling studies, perturbations in membrane voltage generated by putative bAPs with peak depolarisation $\mathrm{V}_{\mathrm{bAP} \text {, max }}$ are modelled as the sum of a large, rapid rise (with a time constant of $\tau_{\mathrm{bAP}, \mathrm{f}}=3 \mathrm{~ms}$ ) and a smaller, slower decay (with a time constant of $\tau_{\mathrm{bAP}, \mathrm{s}}$ ) according to Eq. 3 . The relative contribution of each exponential term is dictated by $\mathrm{I}_{\mathrm{bAP}, \mathrm{f}}=0.75$ and $\mathrm{I}_{\mathrm{bAP}, \mathrm{s}}=0.25[18,48]$.

$$
b A P(t)=V_{b A P, \max }\left(I_{b A P, f} \exp ^{\left(t_{0}-t / \tau_{b A P, f}\right)}+I_{b A P, s} \exp ^{\left(t_{0}-t / \tau_{b A P, s}\right)}\right)
$$

The local Calcium concentration $\left[\mathrm{Ca}^{2+}\right]$ in each dendritic spine is determined by influx through NMDAr - which is directly proportional to the EPSP generated by this receptor - and passive decay with a time constant $\tau_{\mathrm{Ca}}$ according to Eq. 4 [57].

$$
\begin{aligned}
& C a_{N M D A}=-g_{N M D A, C a} P_{N M D A} G_{N M D A}\left(V_{N}-E_{C a}\right) \\
& \frac{d\left[C a^{2+}\right]}{d t}=C a_{N M D A}-\frac{\left[C a^{2+}\right]}{\tau_{C a}}
\end{aligned}
$$

All relevant parameters in the neuron model are fitted to recent data from electrophysiological and fluorescent imaging studies of dendritic spines on CA1 pyramidal neurons during synaptic activation and action potential generation [48]. Maximum excitatory conductance values are chosen to match experimental recordings which indicate that an AMPAr mediated depolarisation of $\sim 10 \mathrm{mV}$ is generated in the spine by activation of a single synapse (giving $g_{A M P A}=23.5 \mathrm{pS}$ ); NMDAr mediated depolarisation of $\sim 5 \mathrm{mV}$ is generated in the spine by activation of a single synapse in the absence of extracellular $\mathrm{Mg}^{2+}$ (giving $g_{N M D A}=3.35 \mathrm{pS}$ ); and bAPs generate a maximum depolarisation of $\mathrm{V}_{b A P, \max }=67 \mathrm{mV}$ in the spine unless stated otherwise $[64,95,96]$. Maximum $\left[\mathrm{Ca}^{2+}\right]$ conductance of the NMDA receptor is set at $g_{N M D A, C a}=0.159 \mathrm{pS}$ to generate a peak $\left[\mathrm{Ca}^{2+}\right]$ concentration of $0.17 \mu \mathrm{M}$ from a single synaptic input at resting membrane potential, and adjusted when the temporal profile of NMDAr activation is manipulated in other simulations to provide the same peak $\left[\mathrm{Ca}^{2+}\right]$ concentration. The decay of $\left[\mathrm{Ca}^{2+}\right]$ proceeds with a time constant of $\tau_{C a}=15 \mathrm{~ms}$, in line with empirical recordings of Calcium dynamics in individual dendritic spines on CA1 pyramidal neurons [57].

\subsection{Plasticity Model}

Transitions between binary high and low weight states for each synaptic input (and vice versa) are governed by a stochastic Markov model in which the probability of making a state change at each timestep is directly proportional to the activity of putative kinase and phophotase pathways respectively. The activity of kinase and phosphotase $\mathrm{p}_{\mathrm{P} / \mathrm{D}}$ above equilibrium values $\mathrm{p}_{\mathrm{P} / \mathrm{D} 0}$ are primarily dictated by modified Hill functions $\sigma_{\mathrm{P}, \mathrm{D}}$ inspired by recent advances in systems biology [49, 50]. These incorporate an effective threshold for activation $\beta_{\mathrm{P}, \mathrm{D}}$ below which kinase and phosphotase activity is effectively buffered and above which activation follows standard formalism (Eq. 5). Hill co-efficients and dissociation constants are set in accordance with previous modelling studies [41?, 44] and the results presented here are robust to significant changes in these parameter values provided that $\mathrm{HN}_{\mathrm{P}}>$ $\mathrm{HN}_{\mathrm{D}}$, negating the need for extensive parameter fitting (data not shown). 
In accordance with previous models, the modified Hill functions operate on local peaks in Calcium concentration, such that step changes in kinase and phosphotase activation occur in any timestep that the first derivative of NMDAr-[Ca $\left.{ }^{2+}\right]$ dynamics is equal to zero and the second derivative is negative [48, MORE?]. The magnitude of these step changes is dictated by the positive constant $\mathrm{k}_{\mathrm{P} / \mathrm{D}}$ and competitive interactions between kinase and phosphotase pathways are incorporated by subtracting the product of kinase activity and a positive constant $\mathrm{k}_{\mathrm{I}}$ from phosphotase activity during step changes. Between local peaks in NMDAr- $\left[\mathrm{Ca}^{2+}\right]$, kinase and phosphotase activity decays towards equilbirium levels with individual time constants $\tau_{\mathrm{P} / \mathrm{D}}$.

The continuous probability of transition between high and low conductance states, and vice versa, in a Markov model is then directly proportional to the instantaneous value of putative kinase and phosphotase activity at each time step. Equilibrium values are subsequently set such that the relative occupation of high and low weight states at rest corresponds to that observed experimentally, $\mathrm{f}_{\mathrm{P} 0}=79 \%$ of synapses occupying the low weight state and $\mathrm{f}_{\mathrm{D} 0}=21 \%$ occupying the high weight state at the start of each simulation [37]. In line with empirical measurements regarding the relative conductance of potentiated and depressed CA3-CA1 synapses, we set the relative strength of high and low weight states as $\mathrm{w}_{\mathrm{P}}=2$ and $\mathrm{w}_{\mathrm{D}}=0.66$ respectively [REFS]. The overall change in putative fEPSP generated by various stimulation protocols can then be assessed by comparing the frequency of synaptic inputs occupying high and low weight states ( $f_{P}$ and $f_{D}$ respectively) at the start and end of each simulation according to Eq. 5 .

$$
\begin{aligned}
& \frac{d p_{P, D}}{d t}=-\frac{\left(p_{P, D}-p_{P 0, D 0}\right)}{\tau_{P, D}} \\
& \left.\begin{array}{l}
\frac{d p_{P}}{d t}=\frac{d p_{P}}{d t}+k_{P} \sigma_{P} \\
\frac{d p_{D}}{d t}=\frac{d p_{D}}{d t}+k_{D} \sigma_{D}-k_{I} \sigma_{P}
\end{array}\right\} \quad \text { if } \frac{d C a}{d t}=0 \cap \frac{d^{2} C a}{d t^{2}}<0 \\
& \sigma_{P, D}=\frac{\left\|C a^{2+}-\beta_{P, D}\right\|^{H N_{P, D}}}{H C_{P, D}+\left\|C a^{2+}-\beta_{P, D}\right\|^{H N_{P, D}}} \\
& \Delta \mathrm{fEPSP}=\frac{\sum f_{P} N w_{P}+f_{D} N w_{D}}{\sum f_{P 0} N w_{P}+f_{D 0} N w_{D}}
\end{aligned}
$$

\subsection{Simulation Details}

During spike pairing stimulation protocols, values of $\Delta \mathrm{t}$ describe the temporal offset between the peak of a single input EPSP and that of a single bAP. In triplet pairing protocols, values of $\Delta t$ describe the temporal offset between the peak of a single input EPSP and the second of two bAPs that are separated by a constant offset of $10 \mathrm{~ms}$. Stimulation frequency in each case is measured as the inverse of temporal offset between the peak of successive input EPSPs.

In tetanic stimulation protocols with stochastic post-synaptic activity, periodic pre-synaptic EPSPs are followed with a probability of $22.2 \%$ (corresponding to the ratio of 200 post-synaptic action potentials to 900 volleys of pre-synaptic field stimulation observed by Wittenberg and Wang [29]) by a single bAP with temporal offset drawn from a random distribution with $\mu=6.2 \mathrm{~ms}$ and $\sigma=4 \mathrm{~ms}$.

The theta-burst stimulation (TBS) protocol consists of delivering ten bursts of four spikes with $10 \mathrm{~ms}$ inter-spike interval and 200ms inter-burst interval either solely pre-synaptically, or simultaneously preand post- synaptically. The depolarisation protocol consists of delivering 100 stimulation pulses at a constant frequency of $2 \mathrm{~Hz}$ while the membrane voltage of the dendritic spine is clamped at a constant value.

All dynamic values are calculated at each $0.1 \mathrm{~ms}$ timestep using Euler integration, and all simulations are performed using MATLAB. 


\section{Acknowledgements}

The authors would like to thank Adam Barrett for useful discussions during the preparation of this manuscript, as well as Samuel Wang, Gayle Wittenberg and Guoqiang Bi for providing experimental data.

\begin{tabular}{|c|c|c|}
\hline Notation & Parameter & Value \\
\hline $\mathrm{C}_{\mathrm{m}}$ & Membrane Capacitance & $1 \mu \mathrm{F}$ \\
\hline $\mathrm{g}_{\mathrm{L}}$ & Leak conductance & $0.1 \mathrm{pS}$ \\
\hline $\mathrm{A}_{\text {spine }}$ & Spine surface area & $17.5 \times 10^{-8} \mathrm{~cm}^{2}$ \\
\hline $\mathrm{g}_{\mathrm{AMPA}}$ & Peak AMPA conductance & $23.5 \mathrm{pS}$ \\
\hline $\mathrm{g}_{\mathrm{NMDA}}$ & Peak NMDA conductance & $3.35 \mathrm{pS}$ \\
\hline$\tau_{\mathrm{AMPA}}$ & AMPA time constant & $5.26 \mathrm{~ms}$ \\
\hline$\tau_{\mathrm{NMDA}, \mathrm{f}}$ & Fast NMDA time constant & $1.485 \mathrm{~ms}$ \\
\hline $\mathrm{E}_{\mathrm{L}}$ & Leak reversal potential & $-65 \mathrm{mV}$ \\
\hline $\mathrm{E}_{\mathrm{AMPA}}$ & AMPA reversal potential & $0 \mathrm{mV}$ \\
\hline $\mathrm{E}_{\mathrm{NMDA}}$ & NMDA reversal potential & $0 \mathrm{mV}$ \\
\hline$\left[\mathrm{Mg}^{2+}\right]$ & Extracellular Magnesium concentration & $1 \mathrm{mM}$ \\
\hline $\mathrm{I}_{\mathrm{bAP}, \mathrm{f}}$ & Contribution of fast bAP time constant & 0.75 \\
\hline $\mathrm{I}_{\mathrm{bAP}, \mathrm{s}}$ & Contribution of slow bAP time constant & 0.25 \\
\hline$\tau_{\mathrm{bAP}, \mathrm{f}}$ & Fast bAP time constant & $3 \mathrm{~ms}$ \\
\hline $\mathrm{g}_{\mathrm{NMDA}, \mathrm{Ca}}$ & NMDAr Calcium conductance & $0.159 \mathrm{pS}$ \\
\hline $\mathrm{E}_{\mathrm{Ca}}$ & Calcium reversal potential & $120 \mathrm{mV}$ \\
\hline$\tau_{\mathrm{Ca}}$ & Time constant of Calcium decay & $15 \mathrm{~ms}$ \\
\hline $\mathrm{P}_{\mathrm{P} 0}$ & Kinase activity / transition probability at rest & $3.22 \times 10^{-6}$ \\
\hline $\mathrm{P}_{\mathrm{D} 0}$ & Phosphotase activity / transition probability at rest & $7.89 \times 10^{-6}$ \\
\hline$\tau_{\mathrm{P}}$ & Kinase time constant & $50 \mathrm{~ms}$ \\
\hline$\tau_{\mathrm{D}}$ & Phosphotase time constant & $2000 \mathrm{~ms}$ \\
\hline $\mathrm{k}_{\mathrm{P}}$ & Kinase activation constant & 0.02 \\
\hline $\mathrm{k}_{\mathrm{D}}$ & Phosphotase activation constant & $4 \times 10^{-4}$ \\
\hline $\mathrm{k}_{\mathrm{I}}$ & Competition constant & 0.2 \\
\hline $\mathrm{HC}_{\mathrm{P}}$ & Kinase dissociation constant & 2 \\
\hline $\mathrm{HC}_{\mathrm{D}}$ & Phosphotase dissociation constant & 2 \\
\hline $\mathrm{HN}_{\mathrm{P}}$ & Hill number of kinase activation & 4 \\
\hline $\mathrm{HN}_{\mathrm{D}}$ & Hill number of phosphotase activation & 3 \\
\hline $\mathrm{N}$ & Total number of synaptic inputs & 10,000 \\
\hline $\mathrm{f}_{\mathrm{P} 0}$ & Initial frequency of synapses in high weight state & 0.21 \\
\hline$f_{D 0}$ & Initial frequency of synapses in low weight state & 0.79 \\
\hline $\mathrm{W}_{\mathrm{P}}$ & Putative strength of high weight state & 2 \\
\hline $\mathrm{w}_{\mathrm{D}}$ & Putative strength of low weight state & 0.66 \\
\hline
\end{tabular}

Table 1: Parameter definitions and values used throughout the simulations presented

\section{References}

[1] Hebb DO: The Organisation of Behaviour. Wiley Press, New York (1949)

[2] Martin SJ, Grimwood PD, Morris RGM (2000) Synaptic plasticity and memory: an evaluation of the hypothesis. Annual Review of Neuroscience 23: 649-711

[3] Whitlock JR, Heynen AJ, Shuler MG, Bear MF (2006) Learning induces long-term potentiation in the hippocampus. Science 313: 1093-1097

[4] Lomo T, Bliss TV (1973) Long-lasting potentiation of synaptic transmission in the dentate area of the anaesthetized rabbit following stimulation of the perforant path. Journal of Physiology 232: 331356

[5] Dudek SM, Bear MF (1992) Homosynaptic long-term depression in area CA1 of hippocampus and effects of N-methyl-D-aspartate receptor blockade. PNAS 89: 4363-7 
[6] Malenka RC, Nicoll RA (1999) Long-term potentiation - a decade of progress? Science 285: 18701874

[7] Citri A, Malenka RC (2008) Synaptic plasticity: multiple forms, functions, and mechanisms. Neuropsychopharmacology 33: 18-41

[8] Neves G, Cooke SF, Bliss TVP (2008) Synaptic plasticity, memory and the hippocampus: a neural network approach to causality. Nature Reviews Neuroscience 9: 65-75

[9] Malenka RC, Bear MF (2004) LTP and LTD: an embarrassment of riches. Neuron 44: 5-21

[10] Cooke SF, Bliss TVP (2006) Plasticity in the human central nervous system. Brain 129: 16591673

[11] Bliss T, Collingridge G, Morris R (2007)Synaptic plasticity in the hippocampus. In: Andersen P, Morris R, Amaral D, Bliss T, O’Keefe J, editors. The Hippocampus Book. Oxford University Press, pp. 343-474

[12] Nelson SB, Turrigiano GG (2008) Strength through diversity. Neuron 60: 477 - 482

[13] Lisman J (1989) A mechanism for the Hebb and the anti-Hebb processes underlying learning and memory. PNAS 86: 9574-9578

[14] Artola A, Singer W (1993) Long-term depression of excitatory synaptic transmission and its relationship to long-term potentiation. Trends in Neuroscience 16: 480-487

[15] Yang SN, Tang YG, Zucker R (1999) Selective induction of LTP and LTD by post-synaptic $\left[\mathrm{Ca}^{2+}\right]_{\mathrm{i}}$ elevation. Journal of Neurophysiology 81: 781-787

[16] Cormier RJ, Greenwood AC, Connor JA (2001) Bidirectional synaptic plasticity correlated with the magnitude of dendritic Calcium transients above a threshold. Journal of Neurophysiology 85, 399406

[17] Mizuno T, Kanazawa I, Sakurai M (2001) Differential induction of LTP and LTD is not determined solely by instantaneous Calcium concentration: an essential involvement of a temporal factor. European Journal of Neuroscience 14: 701 - 708

[18] Shouval HZ, Bear MF, Cooper LN (2002) A unified model of NMDA receptor-dependent bidirectional synaptic plasticity. PNAS 99: 10831-1083

[19] Sjostrom PJ, Nelson SB (2002) Spike timing, calcium signals and synaptic plasticity. Current Opinion in Neurobiology 12: 305-314

[20] Buchanan KA, Mellor JR (2010) The activity requirements for spike timing-dependent plasticity in the hippocampus. Frontiers in Synaptic Neuroscience 2: 11

[21] Malenka RC, Kauer JA, Perkel DJ, Mauk MD, Kelly PT, Nicoll RA, Waxham MN (1989) An essential role for postsynaptic calmodulin and protein kinase activity in long-term potentiation. Nature 340: 554-557

[22] Malinow R, Schulman H, Tsien RW (1989) Inhibition of postsynaptic PKC or CaMKII blocks induction but not expression of LTP. Science 245: 862-866

[23] Hanson PI, Schulman H (1992) Neuronal Ca2+ / Calmodulin-dependent protein kinase. Annual Review of Biochemistry 61: 559-601

[24] Mulkey RM, Herron CE, Malenka RC (1993) An essential role for protein phosphotases in hippocampal long-term depression. Science 261: 1104-1107 
[25] Lee H-K, Barbarosie M, Kameyama K, Bear MF, Huganir RL (2000) Regulation of distinct AMPA receptor phosphorylation sites during bidirectional synaptic plasticity. Nature 405: 955-959

[26] Bi G-Q, Poo M-M (1998) Synaptic Modifications in Cultured Hippocampal Neurons: Dependence on Spike Timing, Synaptic Strength, and Postsynaptic Cell Type. Journal of Neuroscience 77: 551-555

[27] Debanne D, Gähwiler BH, Thompson SM (1998) Long-term synaptic plasticity between pairs of individual CA3 pyramidal cells in rat hippocampal slice cultures. Journal of Physiology 507: 237-247

[28] Pike FG, Meredith RM, Olding AW, Paulsen O (1999) Postsynaptic bursting is essential for 'Hebbian' induction of associative long-term potentiation at excitatory synapses in rat hippocampus. Journal of Physiology 518: 571-6

[29] Wittenberg GM, Wang S-SH (2006) Malleability of spike-timing-dependent plasticity at the CA3CA1 synapse. Journal of Neuroscience 26: 6610-6617

[30] Buchanan KA, Mellor JR (2007) The development of synaptic plasticity induction rules and the requirement for postsynaptic spikes in rat hippocampal CA1 pyramidal neurones. Journal of Physiology 585: 429-445

[31] Caporale N, Dan Y (2008) Spike timing-dependent plasticity: a Hebbian learning rule. Annual Reviews in Neuroscience 31: 25-46

[32] Shouval HZ, Wang SS-H and Wittenberg GM (2010) Spike timing dependent plasticity: a consequence of more fundamental learning rules. Frontiers in Computational Neuroscience 4: 19

[33] Nishiyama M, Hong K, Mikoshiba K, Poo MM, Kato K (2000) Calcium stores regulate the polarity and input specificity of synaptic modification. Nature 408: 584-588

[34] Meredith RM, Floyer-Lea AM, Paulsen O (2003) Maturation of Long-Term Potentiation Induction Rules in Rodent Hippocampus: Role of GABAergic Inhibition. Journal of Neuroscience 23: 1114211146

[35] Sjöström PJ, Turrigiano GG, Nelson SB (2001) Rate, Timing, and Cooperativity Jointly Determine Cortical Synaptic Plasticity. Neuron 32: 1149-1164

[36] Petersen CCN, Malenka RC, Nicoll RA, Hopfield JJ (1998) All-or-none potentiation at CA3-CA1 synapses. PNAS 95: 4732-4737

[37] O'Connor DH, Wittenberg GM, Wang SSH (2005) Graded bidirectional synaptic plasticity is composed of switch-like unitary events. PNAS 102: 9679-9684

[38] O'Connor DH, Wittenberg GM, Wang SSH (2005) Dissection of bidirectional synaptic plasticity into saturable unidirectional processes. Journal of Neurophysiology 94: 1565-1573

[39] Bagal AA, Kao J, Tang C-M, Thompson SM (2005) Long-term potentiation of exogenous glutamate responses at single dendritic spines. PNAS 102: 14434-14439

[40] Fukunaga K, Muller D, Ohmitsu M, Bako’ E, DePaoli-Roach AA, Miyamoto E (2000) Decreased protein phosphatase $2 \mathrm{~A}$ activity in hippocampal long-term potentiation. Journal of Neurochemistry 74 : 807-817

[41] Zhabotinsky AM (2000) Bistability in the Ca2+ / Calmodulin-Dependent Protein KinasePhosphatase System. Biophysical Journal 79: 2211-2221

[42] Karmarkar UR, Buonomano DV (2002) A model of spike-timing dependent plasticity: one or two coincidence detectors? Journal of Neurophysiology 88: 507-513

[43] Yeung LC, Shouval HZ, Blais BS, Cooper LN (2004) Synaptic homeostasis and input selectivity follow from a calcium-dependent plasticity model. PNAS 101: 14943-14948 
[44] Abarbanel HDI, Talathi SS, Gibb L, Rabinovich MI (2005) Synaptic plasticity with discrete state synapses. Physical Review E 72, 031914

[45] Rubin JE, Gerkin RC, Bi G-Q, Chow CC (2005) Calcium Time Course as a Signal for SpikeTiming-Dependent Plasticity. Journal of Neurophysiology 93: 2600-2613

[46] Graupner M, Brunel N (2007) STDP in a bistable synapse model based on CaMKII and associated signalling pathways. PLoS Computational Biology 3(11): e221

[47] Graupner M, Brunel N (2010) Mechanisms of induction and maintenance of spike-timing dependent plasticity in biophysical synapse models. Frontiers in Computational Neuroscience 4: 136

[48] Rackham OJL, Tsaneva-Atanasova K, Ganesh A, Mellor JR (2010) A Ca ${ }^{2+}$-based computational model for NMDA receptor-dependent synaptic plasticity at individual post-synaptic spines in the hippocampus. Frontiers in Synaptic Neuroscience 2: 31

[49] Morgan DO (2007) The cell cycle: principles of control. Sunderland, MA: New Science Press

[50] Buchler NE, Cross FR (2009) Protein sequestration generates a flexible ultrasensitive response in a genetic network. Molecular Systems Biology 5: 272

[51] Krug M, Lossner B, Ott T (1984) Anisomycin blocks the late phase of long-term potentiation in the dentate gyrus of freely moving rats. Brain Research Bulletins 13: 39-42

[52] Nguyen PV, Abel T, Kandel ER (1994) Requirement of a critical period of transcription for induction of a late phase of LTP. Science 165: 1104-1107

[53] Frey U, Morris RG (1997) Synaptic tagging and long-term potentiation. Nature 385: 533-536

[54] Stuart GJ, Sakmann B (1994) Active propagation of somatic action potentials into neocortical pyramidal cell dendrites. Nature 367: 69-72

[55] Magee JC, Johnston D (1997) A Synaptically Controlled, Associative Signal for Hebbian Plasticity in Hippocampal Neurons. Science 275: 209-213

[56] Larkum ME, Zhu JJ, Sakmann B (2001) Dendritic mechanisms underlying the coupling of the dendritic with the axonal action potential initiation zone of adult rat layer 5 pyramidal neurons. Journal of Physiology 533: 447-466

[57] Sabatini BL, Oertner TG, Svoboda K (2002) The life cycle of $\mathrm{Ca}^{2+}$ ions in dendritic spines. Neuron 33: 439-452

[58] Christie BR, Magee JC, Johnston D (1996) The role of dendritic action potentials and $\mathrm{Ca}^{2+}$ influx in the induction of homosynaptic long-term depression in hippocampal CA1 pyramidal neurons. Learning and Memory 3: 160 - 169

[59] Fan Y, Fricker D, Brager DH, Chen X, Lu H-C, Chitwood RA, Johnston D (2005) Activitydependent decrease of excitability in rat hippocampal neurons through increases in $\mathrm{I}_{\mathrm{h}}$. Nature Neuroscience 8: 1542 - 1551

[60] Larson J, Wong D, Lynch G (1986) Patterned stimulation at the theta frequency is optimal for the induction of hippocampal long-term potentiation. Brain Research 368: 347-350

[61] Ngezahayo A, Schachner M, Artola A (2000) Synaptic Activity Modulates the Induction of Bidirectional Synaptic Changes in Adult Mouse Hippocampus. Journal of Neuroscience 20: 2451-2458

[62] Frick A, Magee J, Johnston D (2004) LTP is accompanied by an enhanced local excitability of pyramidal neuron dendrites. Nature Neuroscience 7: 126-135 
[63] Johnston D, Christie BR, Frick A, Gray R, Hoffman DA, Schexnayder LK, Watanabe S, Yuan L-L (2003) Active dendrites, potassium channels and synaptic plasticity. Philosophical Transactions of the Royal Society B 358: 667-674

[64] Canepari M, Djurisic M, Zecevic D (2007) Dendritic signals from rat hippocampal CA1 pyramidal neurons during coincident pre- and post- synaptic activity: a combined voltage- and calcium- imaging study. Journal of Physiology 580: 463-484

[65] Sjostrom PJ, Hausser M (2006) A cooperative switch determines the sign of synaptic plasticity in distal dendrites of neocortical pyramidal neurons. Neuron 51:227-238

[66] Urakubo H, Honda M, Froemke RC, Kuroda S (2008) Requirement of an allosteric kinetics of NMDA receptors for spike-timing dependent plasticity. Journal of Neuroscience 28: 3310-3323

[67] Lisman J, Spruston N (2005) Postsynaptic depolarisation requirements for LTP and LTD: a critique of spike-timing dependent plasticity. Nature Neuroscience 8: 839-841

[68] Erreger K, Dravid SM, Banke TG, Wyllie DJA, Traynelis SF (2005) Subunit-specific gating controls rat NR1/NR2A and NR1/NR2B NMDA channel kinetics and synaptic signalling profiles. Journal of Physiology 563: 345-358

[69] Perez-Otano I, Ehlers MD (2005) Homeostatic plasticity and NMDA receptor trafficking. Trends in Neurosciences 28: 229-238

[70] Klimesch W, Doppelmayr M, Russegger H, Pachinger T (1996) Theta band power in the human scalp EEG and the encoding of new information. Neuroreport 7: 1235-1240

[71] Jeewajee A, Lever C, Burton S, O'Keefe J, Burgess N (2008) Environmental Novelty is Signaled by Reduction of the Hippocampal Theta Frequency. Hippocampus 18: 340-348

[72] Duzel E, Penny WD, Burgess N (2010) Brain oscillations and memory. Current Opinion in Neurobiology 20: 143-149

[73] Fell J, Ludowig E, Staresina BP, Wagner T, Kranz T, Elger CE, Axmacher N (2011) Medial temporal theta/alpha power enhancement precedes successful memory encoding: evidence based on intracranial EEG. Journal of Neuroscience 31: 5392-5397

[74] Lisman JE (1997) Bursts as a unit of neural information: making unreliable synapses reliable. Trends in Neurosciences 20: $38-43$

[75] Harris KD, Hirase H, Leinekugel X, Henze DA, Buzsaki G (2001) Temporal interaction between single spikes and complex spike bursts in hippocampal pyramidal cells. Neuron 32: 141-149

[76] Watt AJ, Sjostrom PJ, Hausser M, Nelson SB, Turrigiano GG (2004) A proportional but slower NMDA potentiation follows AMPA potentiation in LTP. Nature Neuroscience 7: 518-524

[77] Sjöström PJ, Turrigiano GG, Nelson SB (2003) Neocortical LTD via coincident activation of presynaptic NMDA and cannabinoid receptors. Neuron 39: 641-654

[78] Bender VA, Bender KJ, Brasier DJ, Feldman DE (2006) Two coincidence detectors for spike timing-dependent plasticity in somatosensory cortex. Journal of Neuroscience 26: 4166-77

[79] Nevian T, Sakmann B (2006) Spine Ca2+ signaling in spike-timing-dependent plasticity. Journal of Neuroscience 26: 11001-13

[80] Rodríguez-Moreno A, Paulsen O (2008) Spike timing-dependent long-term depression requires presynaptic NMDA receptors. Nature Neuroscience 11: 744-5

[81] Dudman JT, Tsay D, Siegelbaum SA (2007) A role for synaptic inputs at distal dendrites: instructive signals for hippocampal long-term plasticity. Neuron 56: 866-879 
[82] Kampa BM, Letzkus JJ, Stuart GJ (2007) Dendritic mechanisms controlling spike-timing dependent plasticity. Trends in Neuroscience 30: 456-463

[83] Losonczy A, Makara JK, Magee JC (2008) Compartmentalised dendritic plasticity and input feature storage in neurons. Nature 452: 436-441

[84] Legenstein R, Maass W (2011) Branch-specific plasticity enables self-organisation of nonlinear computation in single neurons. Journal of Neuroscience 31: 10787-10802

[85] Desai NS (2003) Homeostatic plasticity in the CNS: synaptic and intrinsic forms. Journal of Physiology, Paris 97: 391-402

[86] Buonomano DV (2005) A Learning Rule for the Emergence of Stable Dynamics and Timing in Recurrent Networks. Journal of Neurophysiology 94: 2275-2283

[87] Lazar A, Pipa G, Triesch J (2009) SORN: a self-organizing recurrent neural network. Frontiers in Computational Neuroscience 3:23

[88] Buzsáki G (2010) Neural Syntax: Cell Assemblies, Synapsembles, and Readers. Neuron 68: 362385

[89] Fiete IR, Senn W, Wang C, Hahnloser RHR (2010) Spike time-dependent plasticity and heterosynaptic competition organize networks to produce long scale-free sequences of neural activity. Neuron 65: 563-576

[90] Bush D, Philippides A, Husbands P, O’Shea M (2010) Dual coding with STDP in an autoassociative network model of the hippocampus. PLoS Computational Biology 6: e1000839

[91] Harris KM, Kater SB (1994) Dendritic spines: Cellular specialisations imparting both stability and flexibility to synaptic function. Annual Reviews in Neuroscience 17: 341-371

[92] Destexhe A, Mainen Z, Sejnowski T (1994) Synthesis of models for excitable membranes, synaptic transmission and neuromodulation using a common kinetic formalism. Journal of Computational Neuroscience 1: 195-230

[93] Dayan P, Abbott LF (2001) Theoretical Neuroscience. London: MIT Press. pp180-183.

[94] Jahr CE, Stevens CF (1990) A quantitative description of NMDA receptor-channel kinetic behaviour. Journal of Neuroscience 10: 1830-1837

[95] Palmer LM, Stuart GJ (2009) Membrane potential changes in dendritic spines during action potentials and synaptic input. Journal of Neuroscience 29: 6897-6903

[96] Fernandez de Sevilla D, Fuenzalida M, Porto Pazos AB, Buno W (2007) Selective shunting of the NMDA EPSP component by the slow afterhyperpolarisation in rat CA1 pyramidal neurons. Journal of Neurophysiology 97: 3242-3255 